\title{
ELEMENTARY ABELIAN OPERATOR GROUPS AND ADMISSIBLE FORMATIONS
}

\author{
FLETCHER GROSS \\ (Received 28 August 1981) \\ Communicated by D. E. Taylor
}

\begin{abstract}
Suppose the elementary abelian group $A$ acts on the group $G$ where $A$ and $G$ have relatively prime orders. If $C_{G}(a)$ belongs to some formation $\mathscr{F}$ for all non-identity elements $a$ in $A$, does it follow that $G$ belongs to $\mathscr{F}$ ? For many formations, the answer is shown to be yes provided that the rank of $A$ is sufficiently large.
\end{abstract}

1980 Mathematics subject classification (Amer. Math. Soc.): 20 D 10.

Suppose $A$ is an elementary abelian $r$-group of order $r^{n}$ which operates on the finite $r^{\prime}$-group $G$. A frequently used method to study this situation is to look at the subgroups $C_{G}(a)$ for the non-identity elements $a \in A$ and to ask whether the structure of these subgroups gives any information about the structure of $G$ as a whole. In this paper, we are interested in the following type of question: If $C_{G}(a) \in \mathscr{F}$, where $\mathscr{F}$ is some "nice" class of groups, for all $a \in A^{\#}$, does it follow that $G \in \mathscr{F}$ ? For this question to have any hope of receiving an affirmative answer, we usually have to exclude certain small values of $n$. Thus we are trying to prove theorems of the following sort: If $C_{G}(a) \in \mathscr{F}$ for all $a \in A^{\#}$ and if $n \geqslant n_{0}$ (where $n_{0}$ depends only on $\mathscr{F}$ ), then $G \in \mathscr{F}$. The solvable signalizer function theorem [5] implies such a result with $\mathscr{F}$ the class of all finite solvable groups and $n_{0}=3$. Another example may be found in [14] where $\mathscr{F}$ is the class of finite nilpotent groups and $n_{0}=3$. We will say that a class $\mathscr{F}$ is admissible provided that such a result is true.

The main thrust of this paper is to determine sufficient conditions for a formation and, in particular, for a subgroup-closed saturated formation to be admissible. If $\mathscr{F}$ is a subgroup-closed saturated formation we find sufficient

Copyright Australian Mathematical Society 1983 
conditions for $\mathscr{F}$ to be admissible in terms of the local formations determining $\mathscr{F}$ (Theorems 3.12 and 3.17). The basis of our results is the following theorem:

Assume $\mathscr{F}$ is an admissible subgroup-closed formation and define $\mathcal{G}$ by

$$
\mathcal{G}=\{G \mid G / K \in \mathscr{F}\} .
$$

(Here $K$ is some specified characteristic subgroup of $G$. Examples of some of the possibilities for $K$ which are covered by this paper are $F(G), Z(G), O_{\pi}(G)$, and $O_{\pi, \pi}(G)$ where $\pi$ is any set of primes.) Then $\mathcal{G}$ is an admissible subgroup-closed formation.

Using this, we may construct many admissible formations. For example, if $K=Z(G)$ and $\mathscr{F}$ consists of all nilpotent groups of class at most $c$, then $\mathcal{G}$ consists of all nilpotent groups of class at most $(c+1)$. In this way, an easy induction yields that

$$
\{G \mid G \text { is a finite nilpotent group of class } \leqslant c\}
$$

is admissible. (For $c=1$, this had been done in [8].)

The groups $G$ we consider need not be solvable. Here, we make use of a simple but rather striking consequence of the classification of all finite simple groups. Namely, if $n \geqslant 2$, we show that any composition factor group of $G$ is isomorphic to a composition factor group of $C_{G}(a)$ for some $a \in A^{\#}$ (Theorem 3.1). All of our theorems may be made independent of the classification by adding the hypothesis that each composition factor group of $G$ is one of the known simple groups. (In this paper, simple does not necessarily imply non-abelian.)

Although most of our results deal with formations, we also prove that certain other classes are admissible. For example, if $\mathscr{F}$ is the class of all finite cyclic groups or if $\mathscr{F}$ consists of all finite groups $G$ such that a Sylow $p$-subgroup of $G$ may be generated by at most $d$ elements (where $p$ and $d$ are fixed), then $\widetilde{F}$ is admissible. Neither of these examples is a formation and the second is not subgroup-closed if $d>1$. On the other hand, we give an example in 3.18 of a subgroup-closed saturated formation which is not admissible. Two subgroupclosed formations whose admissibility is still open are the following:

(1) $\left\{G \mid G^{\prime \prime}=1\right\}$. (More generally, $\left\{G \mid G^{(m)}=1\right\}$.)

(2) $\left\{G \mid x^{p}=1\right.$ for all $\left.x \in G\right\}$ where $p$ is an odd prime.

\section{Notation and introductory results}

All groups considered in this paper are finite. $G^{\#}$ denotes the set of non-identity elements of $G$ while $F(G)$ and $\Phi(G)$ denote the Fitting and Frattini subgroup, respectively, of $G . L_{n}(G)$ is the $n$-th term of the lower central series of $G$, that is, 
$L_{1}(G)=G$ and $L_{n+1}(G)=\left[L_{n}(G), G\right]$. If $G$ is a solvable group, then $l(G)$ denotes its nilpotent length. If $G$ is a nilpotent group, then $\operatorname{cl}(G)$ denotes its class. $\operatorname{Aut}(G)$ is the automorphism group of $G$ while $m(G)$ is the smallest number of elements necessary to generate $G$. If $x$ is a positive real number, then $[x]$ is the largest integer $\leqslant x$. If $V$ is a vector space, then $d(V)$ is its dimension. .

Throughout, $\pi$ denotes a set of primes. If $\pi$ is neither empty nor the set of all primes, then $\pi$ is said to be non-trivial. As usual $\pi^{\prime}$ is the set of primes not belonging to $\pi$. If $G$ is a group, then $K_{\pi}(G)=O_{\pi}(G) O_{\pi^{\prime}}(G)$. Clearly $K_{\pi}(G)=$ $K_{\pi^{\prime}}(G)$. If $\pi$ (or $\pi^{\prime}$ ) consists of a single prime $p$, then we write $K_{p}(G)$. As in [5], a group $G$ is called $\pi$ - separable if each composition factor group of $G$ is either a $\pi$-group or a $\pi^{\prime}$-group. The $\pi$-length, $l_{\pi}(G)$, of the $\pi$-separable group $G$ is defined in [6, page 226]. In our examples, we repeatedly use the fact that in a solvable group $G, l_{\pi}(G) \leqslant[(l(G)+1) / 2]$.

The Greek letter $\Lambda$ is reserved to denote a partition of the set of primes, that is, the members of $\Lambda$ are non-empty sets of primes and each prime belongs to exactly one member of $\Lambda$. If each member of $\Lambda$ is a singleton set, then we call $\Lambda$ the discrete partition. The group $G$ is $\Lambda$-separable if $G$ is $\pi$-separable for each $\pi \in \Lambda$. The subgroup $K_{\Lambda}(G)$ is defined by

$$
K_{\Lambda}(G)=\bigcap_{\pi \in \Lambda} K_{\pi}(G)=\prod_{\pi \in \Lambda} O_{\pi}(G) .
$$

If $\Lambda$ consists of just 2 sets, one of which is $\pi$, then $K_{\Lambda}(G)=K_{\pi}(G)$. If $\Lambda$ is the discrete partition, then $K_{\Lambda}(G)=F(G)$ and a group is $\Lambda$-separable if, and only if, it is solvable.

Following [9], a group $G$ satisfies $C_{\pi}$ if $G$ has exactly one conjugacy class of Hall $\pi$-subgroups. If, in addition, each $\pi$-subgroup of $G$ is contained in a Hall $\pi$-subgroup of $G$, then $G$ satisfies $D_{\pi}$. If $G$ has a normal Hall $\pi$-subgroup (equivalently, if $G / O_{\pi}(G)$ is a $\pi^{\prime}$-group), they we say that $G$ is $\pi$-closed.

Any class $\mathscr{F}$ of groups is to be understood to be closed under isomorphisms (that is, if $G \in \mathscr{F}$ and $G \cong H$, then $H \in \mathscr{F}$ ). The empty class is denoted by $\phi$ while any other classes will be denoted by script letters. A class $\mathscr{F}$ is subgroupclosed if $G \in \mathscr{F}$ and $H \leqslant G$ always implies $H \in \mathscr{F}$. A class $\mathscr{F}$ is admissible if there is a positive integer $n$ such that the following statement is always true.

Suppose $A$ is an elementary abelian group which operates on the group $G$. If $(|A|,|G|)=1, C_{G}(a) \in \mathscr{F}$ for all $a \in A^{\#}$, and $m(A) \geqslant n$, then $G \in \mathscr{F}$.

If $\mathscr{F}$ is admissible, then the smallest positive integer which will work for $n$ is denoted by $n(\tilde{F})$.

A formation $\mathscr{F}$ is a class of groups which is closed under taking homomorphic images and subdirect products. The $\mathscr{F}$-residual of the group $G$ is denoted by $G_{\mathscr{F}}$ 
and is the intersection of all normal subgroups whose factor groups belong to $\mathscr{F} . \mathscr{F}$ is saturated if a group $G$ belongs to $\mathscr{F}$ whenever $G / \Phi(G)$ belongs.

Now suppose that $\mathscr{F}(p)$ is a formation for each prime $p$ and let $\pi=\{p \mid \mathscr{F}(p)$ $\neq \varnothing\}$. Define $e$ by

$$
\mathcal{L}=\left\{G \mid G \text { is a } \pi \text {-group and } G / O_{p^{\prime} p}(G) \in \mathscr{F}(p) \text { for all } p \in \pi\right\} .
$$

Gaschütz showed that $\varrho$ is a saturated formation [10, VI. 7.5]. Conversely, Schmid [11] proved that every saturated formation can be obtained in this way. For the purposes of this paper, better results may sometimes be obtained by working with a slightly different formation. Namely, define $\mathscr{K}$ by

$$
\mathcal{K}=\left\{G \mid G \text { is a } \pi \text {-group and } G / K_{p}(G) \in \mathscr{F}(p) \text { for all } p \in \pi\right\} .
$$

To distinguish between them, we will say that $\mathcal{E}$ is locally defined by $\{\mathscr{F}(p)\}$ while $\mathscr{K}$ is $\mathscr{K}$-generated by $\{\mathscr{F}(p)\}$. It is shown in 2.7 that $\mathscr{K}$ is also a saturated formation. Furthermore, given any saturated formation $\mathscr{F}$, it is always possible to find formations $\mathcal{G}(p)$ such that $\mathscr{F}$ is both locally defined by $\{\mathscr{G}(p)\}$ and also $K$-generated by $\{\mathcal{G}(p)\}$. This does not mean that $\mathcal{E}$ and $\mathcal{K}$ are always the same. For example, if $\mathscr{F}(p)$ is the formation of all $p^{\prime}$-groups for each $p$, then $\mathscr{K}$ is the class of all nilpotent groups while $e$ consists of all solvable groups $G$ satisfying $l_{p}(G) \leqslant 1$ for each $p$. Thus the group $S_{3}$ belongs to $E$ but not to $\mathcal{K}$. It is always true that $\mathcal{K} \subseteq \mathcal{L}$.

We now list some basic results needed later. Most of these are well-known, easily proved, and require no comment.

2.1. The class of all $\pi$-separable groups is closed under taking subgroups, factor groups, direct products, and extensions.

2.2. If $G$ is $\pi$-separable, then $G$ satisfies both $D_{\pi}$ and $D_{\pi^{\prime}}$

Proof. This follows from the Feit-Thompson Theorem [3] and [6, Theorems 6.3.5 and 6.3.6].

2.3. (i) If $H \leqslant G$, then $K_{\Lambda}(G) \cap H \leqslant K_{\Lambda}(H)$.

(ii) If $H \unlhd G$, then $K_{\Lambda}(G) H / H \leqslant K_{\Lambda}(G / H)$.

(iii) $K_{\Lambda}\left(G_{1} \times G_{2}\right)=K_{\Lambda}\left(G_{1}\right) \times K_{\Lambda}\left(G_{2}\right)$.

(iv) $K_{\Lambda}(G) \geqslant \Phi(G)$ and $K_{\Lambda}(G / \Phi(G))=K_{\Lambda}(G) / \Phi(G)$.

(v) $K_{p}(G) \leqslant O_{p^{\prime} p}(G)$ and $O_{p}\left(G / K_{p}(G)\right)=O_{p^{\prime} p}(G) / K_{p}(G)$.

2.4. If $G$ is $\Lambda$-separable, then $C_{G}\left(K_{\Lambda}(G)\right) \leqslant K_{\Lambda}(G)$.

Proof. Let $K=K_{\Lambda}(G)$ and $C=C_{G}(K)$. If $C \neq Z(K)$, then $C / Z(K)$ contains a minimal normal subgroup $H / Z(K)$ of $G / Z(K)$. Since $G$ is $\Lambda$-separable, 
$H / Z(K)$ is a $\pi$-group for some $\pi \in \Lambda$. By $2.2, H$ must have a Hall $\pi$-subgroup $L$. Then, since $[H, Z(K)]=1, H=L \times O_{\pi^{\prime}}(Z(K))$. It follows from this that $L \leq G$. Then $L \leqslant O_{\pi}(G) \leqslant K_{\Lambda}(G)$. Hence $H \leqslant K$ and we have a contradiction.

2.5. Suppose $A$ is an abelian group which operates on the group $G$ with $(|A|,|G|)$ $=1$. Then the following are true:

(i) $G=[G, A] C_{G}(a)$.

(ii) If $H$ is an $A$-invariant normal subgroup of $G$, then $C_{G / H}(a)=C_{G}(a) H / H$.

(iii) If $A$ is not cyclic, then $G=\left\langle C_{G}(a) \mid a \in A^{\#}\right\rangle$.

(iv) If $G$ satisfies $C_{\pi}$, then there is an $A$-invariant Hall $\pi$-subgroup $H$ in $G$ and $C_{H}(a)$ is a Hall $\pi$-subgroup of $C_{G}(a)$ for all $a \in A^{\#}$.

(v) If $G$ is simple, then $A / C_{A}(G)$ is cyclic.

Proof. Section 6.2 of [6] contains (i), (ii), and (iii). The first part of (iv) is well-known and so let $H$ be an $A$-invariant Hall $\pi$-subgroup of $G$. If $p \in \pi$, then $H$ must contain an $A$-invariant Sylow $p$-subgroup $S$ of $G$. Similarly, since $C_{G}(a)$ is $A$-invariant, there is an $A$-invariant Sylow $p$-subgroup $P$ of $C_{G}(a)$. Then there is an $x \in C_{G}(A)$ such that $x^{-1} P x \leqslant S$ [6, Theorem 6.2.2]. But $C_{G}(A)$ is contained in $C_{G}(a)$ and so

$$
x^{-1} P x \leqslant S \cap C_{G}(a) \leqslant H \cap C_{G}(a)=C_{H}(a) .
$$

Hence $C_{H}(a)$ is a $\pi$-subgroup of $C_{G}(a)$ and $C_{H}(a)$ contains a Sylow $p$-subgroup of $C_{G}(a)$ for all $p \in \pi$. This implies that $C_{H}(a)$ is a Hall $\pi$-subgroup of $C_{G}(a)$.

Now (v) depends upon the recently completed classification of all simple groups. For if $B=A / C_{A}(G)$, then $B \leqslant \operatorname{Aut}(G)$ and $(|B|,|G|)=1$. If $G$ is a sporadic group or an alternating group, then this forces $B=1$ (see [1] and [4] for a description of $\operatorname{Aut}(G)$ when $G$ is a sporadic group). If $G$ is a Chevalley group, then it follows from [13] that $B$ is isomorphic to a group of automorphisms of some finite field. Hence $B$ is cyclic in this case.

2.6. Assume that $\mathscr{F}$ is a non-empty formation. Then

(i) If $H \unlhd G$, then $(G / H)_{\mathscr{F}}=G_{\mathscr{F}} H / H$.

(ii) If $\mathscr{F}$ is subgroup-closed and $H \leqslant G$, then $H_{\mathscr{G}} \leqslant H \cap G_{G F}$.

(iii) If $\mathcal{G}=\left\{G \mid G / O_{\pi}(G) \in \mathscr{F}\right\}$, then $\mathcal{G}$ is a formation and $\mathcal{G}$ is subgroup-closed if $\mathscr{F}$ is,

2.7. I. Assume that $\mathscr{F}(p)$ is a formation for each prime $p$. Let $\{\mathscr{F}(p)\}$ locally define $E$ and $K$-generate $\mathcal{K}$. Let $\pi=\{p \mid \mathcal{F}(p) \neq \varnothing\}$.

Then the following are true:

(i) $\mathcal{E}$ and $\mathcal{K}$ are both saturated formations. 
(ii) $\mathscr{E} \mathfrak{K}$.

(iii) If $\mathscr{F}(p)$ is subgroup-closed for each $p \in \pi$, then both $\&$ and $\mathscr{K}$ are subgroupclosed.

(iv) Define $\mathcal{G}(p)$ by $\mathcal{G}(p)=\varnothing$ if $p \notin \pi$ and $\mathcal{G}(p)=\left\{G \mid G / O_{p}(G) \in \mathscr{F}(p)\right\}$ if $p \in \pi$. Then $\mathcal{L}$ is both locally defined and $K$-generated by $\{\mathcal{G}(p)\}$.

II. If $\mathscr{F}$ is a non-empty saturated formation, then there are formations $\mathcal{E}(p)$, one for each prime $p$, such that $\mathscr{F}$ is both locally defined and $K$-generated by $\{\varrho(p)\}$.

Proof. Using 2.3 and [10, VI.7], we easily derive (i), (ii) (since $G / O_{p^{\prime} p}(G)$ is a homomorphic image of $G / K_{p}(G)$ ), and (iii). Now $O_{p}\left(G / O_{p^{\prime} p}(G)\right)=1$ and so $G / O_{p^{\prime} p}(G) \in \mathcal{G}(p)$ if, and only if, $G / O_{p^{\prime} p}(G) \in \mathscr{F}(p)$. Hence $\{\mathcal{G}(p)\}$ locally defines $\mathcal{L}$. Since $O_{p}\left(G / K_{p}(G)\right)=O_{p^{\prime} p}(G) / K_{p}(G)$, we see that $G / K_{p}(G) \in \mathcal{G}(p)$ if, and only if, $G / O_{p^{\prime} p}(G) \in \mathscr{F}(p)$. This implies that $\{\mathscr{S}(p)\} K$-generates $\varrho$. Thus $I$ is proved.

Now any saturated formation is locally defined [12]. Using I(iv), we see that II follows.

The next result follows immediately from the definitions but is very useful in determining whether a class is admissible.

2.8. Let $I$ be a non-empty set and suppose that for each $i \in I$, $\mathscr{F}_{i}$ is an admissible class of groups. Assume that $\left\{n\left(\mathscr{F}_{i}\right) \mid i \in I\right\}$ has an upper bound. If $\mathscr{F}=\bigcap_{i \in I} \mathscr{F}_{i}$, then $\mathscr{F}$ is admissible and $n(\mathscr{F}) \leqslant \sup \left\{n\left(\mathscr{F}_{i}\right) \mid i \in I\right\}$.

Proof. Suppose that $A$ is an elementary abelian group which operates on the group $G$ with $(|A| G \mid)=$,1 . Assume that $C_{G}(a) \in \mathscr{F}$ for all $a \in A^{\#}$. If $m(A) \geqslant$ $n\left(\mathscr{F}_{i}\right)$ for all $i \in I$, then the admissibility of $\mathscr{F}_{i}$ implies that $G \in \mathscr{F}_{i}$ for all $i \in I$. But then $G \in \mathscr{F}$.

For our next results in this introductory section, we present two simple methods of producing new admissible classes from old ones.

2.9. Suppose that $\mathbb{Q}$ is an admissible class of groups such that every group in $\mathbb{Q}$ satisfies $C_{\pi}$. Let $\mathscr{B}$ be an admissible class of groups and define $\mathscr{F}$ by

$$
\mathscr{F}=\{G \mid G \in \mathbb{Q} \text { and a Hall } \pi \text {-subgroup of } G \text { belongs to } \mathscr{B}\} \text {. }
$$

Then $\mathscr{F}$ is admissible and $n(\mathscr{F}) \leqslant \operatorname{Max}\{n(\mathscr{Q}), n(\mathscr{B})\}$.

Proof. Assume $A$ acts on $G,(|A|,|G|)=1, A$ is elementary abelian, $C_{G}(a) \in$ $\mathscr{Y}$ for all $a \in A^{\#}$, and $m(A) \geqslant \operatorname{Max}\{n(\mathbb{Q}), n(\mathscr{B})\}$. Since $C_{G}(a) \in \mathbb{Q}$ and $m(A) \geqslant$ $n(\Theta), G$ must belong to $Q$. Then $G$ satisfies $C_{\pi}$. By $2.5(\mathrm{iv}), G$ has an $A$-invariant 
Hall $\pi$-subgroup $H$ and $C_{H}(a)$ is a Hall $\pi$-subgroup of $C_{G}(a)$. Since $C_{G}(a)$ satisfies $C_{\pi}$ (since $C_{G}(a) \in \mathbb{Q}$ ), $C_{H}(a)$ must belong to $\mathscr{B}$ for all $a \in A^{\#}$. Since $m(A) \geqslant n(\mathscr{B}), H \in \mathscr{B}$. But then $G \in \mathscr{F}$.

REMARK. If $\pi=\{p\}$, then $\mathscr{Q}$ may be the class of all groups.

2.10. Suppose that $T(G)$ is a characteristic subgroup of $G$ for each group $G$. Assume that the following hold:

(i) If $\sigma$ is an isomorphism of $G$ onto $H$, then

$$
T(H)=(T(G))^{\circ} .
$$

(ii) If $H \leqslant G$, then $T(G) \cap H \leqslant T(H)$.

Assume that $Q$ is an admissible subgroup-closed class of groups and define $\Re$ by

$$
\Re=\{G \mid T(G) \in \mathbb{Q}\} .
$$

Then $\mathscr{B}$ is admissible and $n(\mathscr{B}) \leqslant n(\mathbb{Q})$.

Proof. First, note that there are many choices for $T(G)$ that satisfy (i) and (ii). For example, $T(G)$ could be any of the following: $Z(G), F(G), O_{\pi}(G), K_{\Lambda}(G)$, $O_{\pi^{\prime} \pi}(G)$.

Now suppose $A$ acts on $G,(|A|,|G|)=1, A$ is elementary abelian, $C_{G}(a) \in \mathscr{B}$ for all $a \in A^{\#}$, and $m(A) \geqslant n(\mathbb{Q})$. If $H=T(G)$, then $C_{H}(a)=H \cap C_{G}(a) \leqslant$ $T\left(C_{G}(a)\right)$. Since $C_{G}(a) \in \mathscr{B}$ and $Q$ is subgroup-closed, $C_{H}(a) \in \mathbb{Q}$ for all $a \in A^{\#}$. Since $m(A) \geqslant n(a)$, we see that $H \in \mathbb{Q}$ and $G \in \mathscr{B}$.

To illustrate 2.10 , consider groups whose center is the identity. Now if $\mathscr{Q}$ is the identity class, (that is, $G \in \mathbb{U}$ if, and only if, $|G|=1$ ), then $\mathbb{E}$ is subgroup-closed and it follows from 2.5 (iii) that $n(\mathbb{Q})=2$. If

$$
\Re=\{G \mid Z(G)=1\},
$$

then 2.10 implies that $\mathscr{B}$ is admissible and $n(\mathscr{B}) \leqslant 2$.

Virtually all of the admissible classes to be considered later are subgroup-closed. The example just given is an exception. (If $H \leqslant G$ and $Z(G)=1$, it does not follow that $Z(H)=1$.) Another exception is given in 2.12 below. This depends upon the following easy result about modules for elementary abelian groups.

2.11. Let $A$ be an elementary abelian $r$-group and let $F$ be a field of characteristic $\neq r$. Let $s$ be the degree of $\lambda$ over $F$ where $\lambda$ is a primitive $r$-th root of unity in the algebraic closure of $F$. Assume that $V$ is an $F A$-module and that $d\left(C_{V}(a)\right) \leqslant n$ for all $a \in A^{\#}$. If $m(A) \geqslant 1+(n+1) / s$, then $d(V) \leqslant n$. 
Proof. If $U$ is any irreducible $F A$-module and $C_{A}(U) \neq A$, then our assumptions imply that $d(U)=s$. If $a \in A^{\#}$, then $C_{V}(a)$ is the direct sum of irreducible $F A$-submodules. It follows that if $n<s$, then $C_{V}(a)=C_{V}(A)$ for all $a \in A^{\#}$. But then each element of $A$ acts fixed point-freely on $V / C_{V}(A)$. This is impossible if $V \neq C_{V}(A)$ since $A$ is not cyclic $(m(A)>1)$. Hence if $n<s, V=C_{V}(A)=C_{V}(a)$ and so $d(V) \leqslant n$.

Assume now that $n \geqslant s$ and proceed by induction on $n$. If $C_{A}(V) \neq 1$, then $V \leqslant C_{V}(a)$ for some $a \in A^{\#}$ and the result is trivial. Thus assume $C_{A}(V)=1 . V$ must contain a non-trivial irreducible $F A$-submodule $U$. Then $d(U)=s$ and $\left|A / C_{A}(U)\right|=r$. If $B=C_{A}(U)$, then $m(B)=m(A)-1 \geqslant(n+1) / s$. Also $C_{V}(b) \supseteq U$ for all $b \in B^{\#}$. Hence

$$
d\left(C_{V / U}(b)\right)=d\left(C_{V}(b)\right)-d(U) \leqslant n-s .
$$

By induction then, $d(V / U) \leqslant n-s$ and so $d(V) \leqslant n$.

2.12. Let $p$ be a prime, let $d$ be a non-negative integer, and let $\mathscr{F}$ be the class of all groups $G$ such that $m(P) \leqslant d$ where $P$ is a Sylow p-subgroup of $G$. Then $\mathscr{F}$ is admissible and

$$
n(\mathscr{F})= \begin{cases}d+2 & \text { if } p \neq 2 \\ {\left[\frac{d}{2}\right]+2} & \text { if } p=2\end{cases}
$$

Proof. If $d=0$, then the requirement on $P$ is that $P=1$. Since $n$ (\{identity $\})$ $=2$, the result follows from 2.9 in this case. Assume now that $d \geqslant 1$ and that $m=d+2$ if $p$ is odd and $m=[d / 2]+2$ if $p=2$.

Assume that $A$ acts on $G,(|A|,|G|)=1, A$ is an elementary abelian $r$-group, $C_{G}(a) \in \mathscr{F}$ for all $a \in A^{\#}$, and $m(A) \geqslant m$. Then by 2.5 (iv), there is an $A$-invariant Sylow $p$-subgroup $P$ and $C_{P}(a)$ is a Sylow $p$-subgroup of $C_{G}(a)$. Hence $m\left(C_{P}(a)\right) \leqslant d$ for all $a \in A^{\#}$.

Let $V$ be $P / \Phi(P)$ written additively. Then $V$ is a $G F(p) A$-module and $d\left(C_{V}(a)\right) \leqslant d$ for all $a \in A^{\#}$. Let $s$ be the degree of a primitive $r$-th root of unit over $G F(p)$. Then $s \geqslant 1$ and so

$$
1+\frac{d+1}{s} \leqslant 1+(d+1)=m \leqslant m(A) .
$$

if $p \neq 2$. Hence, if $p \neq 2$, it follows from 2.11 that $|P / \Phi(P)| \leqslant p^{d}$ and so $G \in \mathscr{F}$. If $p=2$, then $s$ must be at least 2 . In that case

$$
1+\frac{d+1}{s} \leqslant 1+\frac{d+1}{2} \leqslant\left[\frac{d}{2}\right]+2 \leqslant m(A) .
$$

Hence, again by $2.11,|P / \Phi(P)| \leqslant p^{d}$ and so $G \in \mathscr{F}$. 
So far, we have proved that $\mathscr{F}$ is admissible and that $n(\mathscr{F}) \leqslant m$. To prove that $n(\mathscr{F})$ cannot be any smaller, we construct some examples.

If $p \neq 2$, let $r$ be any prime dividing $p-1(r=2$, for example). Let $G$ be an elementary abelian group of order $p^{d+1}$. Then $\operatorname{Aut}(G)$ contains an elementary abelian $r$-group $A$ of order $r^{d+1}$. Then, if $a \in A^{\#}, C_{G}(a)<G$ and so $m\left(C_{G}(a)\right) \leqslant$ $d$. Thus $C_{G}(a) \in \mathscr{F}$ for all $a \in A^{\#}$ but $G \notin \mathscr{F}$.

If $p=2$, let $n=[d / 2]$ and let $G$ be an elementary abelian group of order $2^{2 n+2}$. Then $\operatorname{Aut}(G)$ contains an elementary abelian 3-group $A$ of order $3^{n+1}$. Since for all $a \in A^{\#}, a$ must act faithfully on $G / C_{G}(a)$, we must have $\left|C_{G}(a)\right| \leqslant$ $2^{2 n}$. Then $C_{G}(a) \in \mathscr{F}$ for all $a \in A^{\#}$ but $G \notin \mathscr{F}$.

REMARK. If $d>1$, then $\mathscr{F}$, the class in the above result, is not subgroup-closed. Nor is $\mathscr{F}$ a formation since $\mathscr{F}$ is not closed under direct products.

\section{The main results}

The next result is a direct consequence of the classification of all simple groups. The theorem could be made independent of the classification by adding the assumption that the composition factor group in question is a known simple group.

3.1. THEOREM. Suppose that $A$ is an abelian but not cyclic group which operates on the group $G$ with $(|A|,|G|)=1$. Then any composition factor group of $G$ occurs as a composition factor group of some $C_{G}(a)$ with $a \in A^{\#}$.

Proof. Replacing $A$ by one of its subgroups, if necessary, we may assume that $A$ is elementary abelian and $m(A)=2$. Let $M$ be a minimal $A$-invariant normal subgroup of $G$. By induction, any composition factor group of $G / M$ occurs as a composition factor group of $C_{G / M}(a)$ for some $a \in A^{\#}$. Now $C_{G / M}(a) \cong$ $C_{G}(a) / C_{M}(a)$ and so the theorem will be proved once we show that any composition factor group of $M$ occurs as a composition factor group of $C_{M}(a)$ for some $a \in A^{\#}$. Thus it suffices to prove the theorem when $M=G$.

Hence $G$ is a minimal normal subgroup of $G A$. Thus, if $G$ is abelian, all composition factor groups of $G$ and of any subgroup of $G$ are the same. Since $G=\left\langle C_{a}(a) \mid a \in A^{\#}\right\rangle$, there is an $a \in A^{\#}$ such that $C_{G}(a) \neq 1$. The theorem now follows.

Assume, therefore, that $G$ is non-abelian. Then

$$
G=S_{1} \times \cdots \times S_{n}
$$


where $S_{1}, \ldots, S_{n}$ are isomorphic, simple, non-abelian groups and $A$ must permute $\left\{S_{1}, \ldots, S_{n}\right\}$ transitively. Since $A$ is abelian, we must have

$$
N_{A}\left(S_{1}\right)=N_{A}\left(S_{2}\right)=\cdots=N_{A}\left(S_{n}\right) .
$$

Thus if $n>1$, there is an $a \in A^{\#}$ such that

$$
\langle a\rangle \cap N_{A}\left(S_{\imath}\right)=1
$$

for all $i$. But then $C_{G}(a)$ is the direct product of $(n /|\langle a\rangle|)$ copies of $S_{1}$ and so certainly the theorem is true in this case.

Finally, assume $n=1$. Then $G$ is a simple group and so by $2.5(\mathrm{v}), A / C_{A}(G)$ is cyclic. Then $C_{A}(G) \neq 1$ and so $C_{G}(a)=G$ for some $a \in A^{\#}$. Thus the theorem is proved.

3.2. Corollary. $n(\mathscr{F}) \leqslant 2$ if $\mathscr{F}$ is any of the following classes: $\{G \mid G$ is solvable $\},\{G|| G \mid=1\},\{G \mid G$ is a $\pi$-group $\},\{G \mid G$ is $\Gamma$-separable $\}$.

Proof. For each of these classes, $G \in \mathscr{F}$ if, and only if, each composition factor group of $G$ belongs to $\mathscr{F}$. The corollary now follows.

More generally, suppose $\subseteq$ is a class of simple groups (and here simple does not necessarily imply non-abelian) and let $\mathscr{F}$ consist of those groups $G$ such that each composition factor group of $G$ belongs to $\mathcal{S}$. Then $\mathscr{F}$ is admissible and $n(\mathscr{F}) \leqslant 2$.

The next result is the main theorem of this paper.

3.3. ThEOREM. Let $\mathscr{F}$ be an admissible subgroup-closed formation. Let $\mathscr{G}=\{G \mid$ $\left.G / K_{\Lambda}(G) \in \mathscr{F}\right\}$. Then $\mathcal{G}$ is an admissible, saturated, subgroup-closed formation and $n(\mathcal{G}) \leqslant n(\mathscr{F})+1$.

Proor. Using 2.3, we easily conclude that $\mathcal{G}$ is a subgroup-closed saturated formation. Assume now that $A$ acts on $G,(|A|,|G|)=1, A$ is elementary abelian, $C_{G}(a) \in \mathcal{G}$ for all $a \in A^{\#}$, and $m(A) \geqslant n(\mathscr{F})+1$. We need to prove that $G \in \mathcal{G}$. Suppose that $G$ is a minimal counterexample. Let $M=G_{\mathcal{G}}$ and $K=G_{\mathscr{g}}$. Then $M$ and $K$ must be non-identity characteristic subgroups of $G$. Let $\pi$ be some member of $\Lambda$ such that $\pi$ contains at least one prime dividing $|M|$. We now proceed in a series of steps.

1. (i) $C_{A}(G)=1$.

(ii) If $H$ is any non-identity $A$-invariant normal subgroup of $G$, then $H \geqslant M$.

(iii) If $H$ is any $A$-invariant proper subgroup of $G$, then $H \in \mathcal{G}$.

(iv) Either $K_{\Lambda}(G)=1$ or $K_{\Lambda}(G)=O_{\pi}(G) \geqslant M$. 
Proof. If $C_{A}(G) \neq 1$, then for some $a \in A^{\#}, G=C_{G}(a) \in \mathcal{G}$. If $1<H \triangleleft G A$ and $H \leqslant G$, then the minimality of $G$ implies that $G / H \in \mathcal{G}$. But then $H \geqslant M$. The minimality of $G$ together with the fact that $\mathcal{G}$ is subgroup-closed imply (iii). Finally, suppose $K_{\Lambda}(G) \neq 1$. Then $K_{\Lambda}(G) \geqslant M$ from (ii). Since $K_{\Lambda}(G)$ is the direct product of a $\pi$-group and a $\pi^{\prime}$-group, it follows from (ii) that $K_{\Lambda}(G)$ is either a $\pi$-group or a $\pi^{\prime}$-group. Since $M$ is not a $\pi^{\prime}$-group, (iv) follows.

\section{2. $C_{G}(M)=Z(M)$.}

Proof. Suppose $C_{G}(M) \neq Z(M)$. then $C_{G}(M)$ is a non-identity $A$-invariant normal subgroup of $G$. Then $C_{G}(M) \geqslant M$ and so $M$ is abelian. Then $M \leqslant F(G)$ $\leqslant K_{\Lambda}(G)$ and so $K_{\Lambda}(G) \neq 1$. This implies that $M \leqslant O_{\pi}(G)=K_{\Lambda}(G)$. Since $G / M \in \mathcal{S}$ and $K=G_{\mathscr{F}}$, we must have $K / M \leqslant K_{\Lambda}(G / M)$. However, $G \notin \mathscr{F}$ and so $K \$ K_{\Lambda}(G)$. It follows from this that $K / M$ is not a $\pi$-group. Since $K$ must be $\pi$-separable (since $K / M \leqslant K_{\Lambda}(G / M)$ ), 2.2 implies that $K$ satisfies $D_{\pi^{\prime}}$. Then there must be an $A$-invariant Hall $\pi^{\prime}$-subgroup $S$ in $K$. Then $S M / M=O_{\pi^{\prime}}(K / M)$. It follows from this that $S M \unlhd G$. Then $G=M N_{G}(S)$. Since $S \neq 1, N_{G}(S) \neq G$. Since $M$ is abelian, $M \cap N_{G}(S)$ is an $A$-invariant normal subgroup of $G$. Then we must have $N_{G}(S) \cap M=1$. Then $C_{G}(M) \cap N_{G}(S)$ is an $A$-invariant normal subgroup of $G$ which does not contain $M$. Hence $C_{G}(M) \cap N_{G}(S)=1$. It now follows that $C_{G}(M)=M$.

3. Let $B=C_{A}(G / M)$. Then

(i) If $a \in A-B$, then $C_{G}(a) K_{\Lambda}(G) / K_{\Lambda}(G) \in \mathscr{F}$.

(ii) $m(B) \geqslant 2$.

Proof. Let $a \in A-B, H=C_{G}(a) M$ and $L=H_{\mathscr{F}}$. Since $a \notin B, H<G$. Then, from (1(iii)), $H \in \mathcal{G}$. Hence $L \leqslant K_{\Lambda}(H)$. Now if $L=1$, then $H \in \widetilde{F}$, and, since $\mathscr{F}$ is subgroup-closed, $C_{G}(a) \in \mathscr{F}$ and (i) follows. Assume now that $L \neq 1$. Then $K_{\Lambda}(H) \neq 1$ and

$$
\left[M, K_{\Lambda}(H)\right] \leqslant M \cap K_{\Lambda}(H) \leqslant K_{\Lambda}(M) \leqslant K_{\Lambda}(G) .
$$

Now if $M \cap K_{\Lambda}(H)=1$, then $K_{\Lambda}(H) \leqslant C_{G}(M) \leqslant M$ which contradicts $K_{\Lambda}(H)$ $\neq 1$. We now see that $K_{\Lambda}(G) \neq 1$. But then $K_{\Lambda}(G)=O_{\pi}(G) \geqslant M$. Now $\left[O_{\pi^{\prime}}(H), M\right]=1$ and so $K_{\Lambda}(H)=O_{\pi}(H)$. Thus $L$ is a $\pi$-group. Since $L=H_{\bar{g}} \leqslant$ $G_{\overline{\mathscr{y}}}=K$ and $K / M \leqslant K_{\Lambda}(G / M)$ (since $G / M \in \mathscr{F}$ ), we must have $L M / M \leqslant$ $O_{\pi}(G / M)$. It follows from this that $L \leqslant O_{\pi}(G)$. This implies that $C_{G}(a) K_{\Lambda}(G) / K_{\Lambda}(G) \in \mathscr{F}$

Now suppose $m(B)<2$. Then $A$ contains a subgroup $B_{0}$ such that $A=B \times B_{0}$ and $m\left(B_{0}\right) \geqslant m(A)-1 \geqslant n(\mathscr{F})$. Since, by (i), $C_{G}(b) K_{\Lambda}(G) / K_{\Lambda}(G) \in \mathscr{F}$ for all $b \in B_{0}^{\#}$, this would imply that $G / K_{\Lambda}(G) \in \mathscr{F}$ and so $G \in \mathcal{G}$. 
4. Let $C=C_{G}(B)$ and $D=C_{\mathscr{F}}$. Then

(i) $G=C M$.

(ii) $C \in \mathcal{G}$.

(iii) If $K_{\Lambda}(G) \neq 1$, then $D \leqslant O_{\pi}(G)$.

(iv) $K_{\Lambda}(G)=1$.

Proof. $B$ centralizes $G / M$ and so $G=C M$. $C_{A}(G)=1$ and so $C<G$. Then, by (1(iii)), $C \in \mathcal{G}$. Therefore, $D \leqslant K_{\Lambda}(C)$. Suppose now that $K_{\Lambda}(G) \neq 1$. Then $K_{\Lambda}(G)=O_{\pi}(G) \geqslant M$. Now $D=O_{\pi}(D) \times O_{\pi^{\prime}}(D), M=\left\langle C_{M}(b) \mid b \in B^{\#}\right\rangle$, and, since $C_{G}(b) \geqslant C, D \leqslant\left(C_{G}(b)\right)_{\mathscr{F}}$ for $b \in B^{\#}$. Since $C_{G}(b) \in \mathcal{G}$, we have $O_{\pi^{\prime}}(D) \leqslant$ $O_{\pi},\left(C_{G}(b)\right)$. This implies that

$$
\left[O_{\pi^{\prime}}(D), C_{M}(b)\right] \leqslant\left[o_{\pi^{\prime}}\left(C_{G}(b)\right), O_{\pi}\left(C_{G}(b)\right)\right]=1 \text {. }
$$

Hence $O_{\pi^{\prime}}(D)$ centralizes $M$. Since $C_{G}(M) \leqslant M \leqslant O_{\pi}(G), O_{\pi^{\prime}}(D)=1$. Then $D$ is a $\pi$-group. But $D<G_{\tilde{y}}=K$ and $K / M \leqslant K_{\Lambda}(G / M)$. It now follows that $D \leqslant$ $O_{\pi}(G)$.

If $K_{\Lambda}(G) \neq 1$, we have just shown that $C O_{\pi}(G) / O_{\pi}(G)$ belongs to $\mathscr{F}_{\mathscr{f}}$. But $G=C M=C O_{\pi}(G)$ and so $G / O_{\pi}(G) \in \mathscr{F}$. Since this would mean that $G \in \mathcal{G}$, we must have $K_{\Lambda}(G)=1$.

5. $M=S_{1} \times \cdots \times S_{n}$ where $S_{1}, \ldots, S_{n}$ are isomorphic, simple, non-abelian groups which are permuted transitively by $C A$. Also, $C_{G}(M)=1$.

Proof. $M$ is a minimal normal subgroup of $G A=M C A$. If $M^{\prime}=1$, then $M \leqslant K_{\Lambda}(G)$. Since $K_{\Lambda}(G)=1, M^{\prime} \neq 1$. (5) now follows. $\left(C_{G}(M)=Z(M)=1\right.$.)

6. Let $B_{1}=N_{B}\left(S_{1}\right)$. Then

(i) $B_{1}=N_{B}\left(S_{k}\right)$ for all $k, 1 \leqslant k \leqslant n$.

(ii) $C_{B}\left(S_{k}\right)=1$ for all $k, 1 \leqslant k \leqslant n$.

(iii) $m\left(B_{1}\right) \leqslant 1$.

(iv) If $b \in B-B_{1}$, then $K_{\Lambda}\left(C_{M}(b)\right)=\left[D, C_{M}(b)\right]=1$.

Proof. $C A$ permutes $\left\{S_{1}, \ldots, S_{n}\right\}$ transitively and $B \leqslant Z(C A)$. It follows from this that $B_{1}=N_{B}\left(S_{K}\right)$ for all $k$ and that $C_{B}\left(S_{1}\right)=C_{B}\left(S_{2}\right)=\cdots=C_{B}\left(S_{n}\right)$. Since $C_{B}(M)$ centralizes both $M$ and $G / M, C_{B}(M) \leqslant C_{A}(G)=1$. Thus (i) and (ii) are proved. Using $2.5(\mathrm{v})$, we obtain (iii).

Now suppose $b \in B-B_{1}$. Then $\langle b\rangle$ permutes $\left\{S_{1}, \ldots, S_{n}\right\}$ semi-regularly. Then $C_{M}(b)$ is the direct product of $(n /|\langle b\rangle|)$ copies of $S_{1}$. Now $S_{1}$ is not $\Lambda$-separable since $K_{\Lambda}(G)=1$. It now follows that $K_{\Lambda}\left(C_{M}(b)\right)=1$. But $C_{G}(b) \geqslant C$ and so $D=C_{G F} \leqslant\left(C_{G}(b)\right)_{\mathscr{F}} \leqslant K_{\Lambda}\left(C_{G}(b)\right)$ since $C_{G}(b) \in \mathcal{G}$. Then $\left[D, C_{M}(b)\right] \leqslant$ $K_{\Lambda}\left(C_{G}(b)\right) \cap C_{M}(b) \leqslant K_{\Lambda}\left(C_{M}(b)\right)=1$. 
7. $D=1$.

Proof. Suppose $D \neq 1$. Then $[D, M] \neq 1$. Then, without loss of generality, we may assume that $\left[D, S_{1}\right] \neq 1$. Since $m(B) \geqslant 2>m\left(B_{1}\right)$ there exists an element $b \in B-B_{1}$. Then, without loss of generality, we may assume that $\langle b\rangle$ transitively permutes $\left\{S_{1}, S_{2}, \ldots, S_{r}\right\}$. Then there is a diagonal subgroup $S$ of $S_{1} \times S_{2}$ $\times \cdots \times S_{r}$ which is a direct factor of $C_{M}(b)$. Then, from (6(iv)), $[D, S]=1$. It follows from this that $D$ normalizes $S_{1} \times S_{2} \times \cdots \times S_{r}$. Now if $\bar{D}$ and $\bar{b}$ denote the permutations induced on $\left\{S_{1}, \ldots, S_{r}\right\}$, then $\langle\bar{b}\rangle$ is an abelian regular group and since $[D, b]=1$, it follows that $\bar{D} \leqslant\langle\bar{b}\rangle$. But $(|D|,|\langle b\rangle|)=1$. Hence $D$ must normalize $S_{k}$ for $1 \leqslant k \leqslant r$. Then, since $D$ centralizes $S$ and $D$ normalizes $S_{1}$, we must have $\left[D, S_{1}\right]=1$.

\section{Contradiction.}

Proof. $A$ must contain a subgroup $A_{1}$ such that $A=A_{1} \times B_{1}$. Then $m\left(A_{1}\right)=$ $m(A)-m\left(B_{1}\right) \geqslant m(A)-1 \geqslant n(\mathscr{F})$. Now $G \notin \mathscr{F}$. then there must be an $a \in A_{1}^{\#}$ such that $C_{G}(a) \notin \mathscr{F}$. But if $a \notin B$, then it follows from (3(i)) (since $K_{\Lambda}(G)=1$ ) that $C_{G}(a) \in \mathscr{F}$. Hence $a \in B \cap A_{1}$. Then $C_{G}(a) \geqslant C$ and so $C_{G}(a)=C_{M}(a) C$. Since $C_{\mathscr{F}}=D=1$, we have $C_{G}(a) / C_{M}(a) \in \mathscr{F}$. Since $C_{G}(a) \in \mathcal{G}$ by hypothesis, we must have

$$
\left(C_{G}(a)\right)_{\mathscr{F}} \leqslant C_{M}(a) \cap K_{\Lambda}\left(C_{G}(a)\right) \leqslant K_{\Lambda}\left(C_{M}(a)\right) .
$$

Since $K_{\Lambda}\left(C_{M}(a)\right)=1$ by $\left(6(\right.$ iv) $)$, we have $C_{G}(a) \in \mathscr{F}$ and so the proof is complete.

The next result, which was proved in [8] and independently by Jones [11], now follows immediately.

3.4. Corollary. For each non-negative integer $k$, let $\Re_{k}$ denote the class of all solvable groups of nilpotent length $\leqslant k$. Then $\mathfrak{X}_{k}$ is admissible and $n\left(\mathfrak{X}_{k}\right)=k+2$.

Proof. $\mathscr{T}_{0}$ is the class of identity groups and so $n\left(\mathscr{R}_{0}\right)=2$. Now let $\Lambda$ be the discrete partition. Then $K_{\Lambda}(G)=F(G)$, and, for $k \geqslant 1, G \in \mathfrak{x}_{k}$ if, and only if, $G / F(G) \in \mathfrak{X}_{k-1}$. Using the theorem and induction on $k$, we obtain $n\left(\mathscr{X}_{k}\right) \leqslant k$ +2 for all $k$. Examples in [8] show that $n\left(\mathscr{N}_{k}\right)$ cannot be less than $k+2$.

REMARKs. $1 . \mathscr{T}_{1}$ is the class of all nilpotent groups and so the corollary includes Ward's result [14]. 
2. By analogy with nilpotent length, one could define a $\Lambda$-length for any $\Lambda$-separable group (that is $K_{0}=1$ and $K_{n+1}(G) / K_{n}(G)=K_{\Lambda}\left(G / K_{n}(G)\right)$. Then the same argument as in 3.4 may be used to prove that $n\left(\left\{G \mid K_{k}(G)=G\right\}\right) \leqslant k$ +2 .

The special case $\pi=\{p\}$ of the next result appears in [15].

3.5. Corollary. If $\pi$ is non-trivial and if $\mathbb{Q}$ is the class of all $\pi$-closed groups, then $\mathbb{Q}$ is admissible and $n(\mathbb{Q})=3$.

Proof. Let $\Lambda=\left\{\pi, \pi^{\prime}\right\}$ and let $\mathscr{F}$ be the class of all $\pi^{\prime}$-groups. Then $n(\mathscr{F})=2$ by 3.2 and $\mathbb{Q}=\left\{G \mid G / K_{\Lambda}(G) \in \mathscr{F}\right\}$. The theorem now yields $n(\mathbb{Q}) \leqslant 3$. Example 1 in $\$ 4$ shows that $n(\Theta)$ cannot be any smaller.

3.6. Corollary. If $\prec$ is a total ordering of the set of all primes and if $\mathbb{Q}$ is the class of all groups which have a Sylow tower of type $\prec$, then $n(\mathbb{Q})=3$.

Proof. Sylow tower of type $\prec$ is defined in [10, VI.6.13] where it is shown that $G \in \mathcal{Q}$ if, and only if, $G$ is $\pi_{i}$-closed for all $i=1,2, \ldots$ and $\pi_{1}, \pi_{2}, \ldots$ are sets of primes depending on $\prec$. Thus $\mathbb{Q}$ is the intersection of classes of the type in the previous corollary. Using that result and 2.8 , we easily obtain the result.

\subsection{THEOREM. Let $\widetilde{\mathcal{F}}$ be an admissible, subgroup-closed formation and let}

$$
\mathcal{G}=\{G \mid G / Z(G) \in \mathscr{F}\} .
$$

Then $\mathcal{G}$ is an admissible, subgroup-closed formation and $n(\mathcal{G}) \leqslant n(\mathscr{\mathscr { F }})+1$.

Proof. It is easily verified that $\mathcal{G}$ is a subgroup-closed formation. Now suppose $A$ acts on $G,(|A|,|G|)=1, A$ is elementary abelian, $C_{G}(a) \in \mathcal{G}$ for all $a \in A^{\#}$, and $m(A) \geqslant n(\mathcal{F})+1$. Assume that $G$ is a minimal example such that $G \notin \mathcal{G}$. Let $M$ be the $\mathcal{G}$-residual of $G$. Then $M \neq 1$.

1. (i) If $H$ is a non-identity $A$-invariant normal subgroup of $G$, then $H \geqslant M$.

(ii) $G_{\mathscr{F}} / M \leqslant Z(G / M)$.

Proof. In (i), $G / H \in \mathcal{G}$ by the minimality of $G$. This implies $H \geqslant M$. Now $G \notin \mathcal{G}$ and so $G \notin \mathscr{F}$. Then $G_{\mathscr{\mathcal { F }}} \geqslant M$ by (i). Now $G / M \in \mathcal{G}$ and so $G_{\tilde{\mathfrak{y}}} / M \leqslant$ $Z(G / M)$.

2. $Z(G)=1$. 
Proof. Suppose $Z(G) \neq 1$. Then $M \leqslant Z(G)$. Then $M$ is an irreducible $A$-module and so $A / C_{A}(M)$ must be cyclic. Then $m\left(C_{A}(M)\right) \geqslant n(\mathscr{F})$. Let $a \in\left(C_{A}(M)\right)^{\mp}$, $C=C_{G}(a)$, and $D=C_{\mathscr{F}}$. Then $C \in \mathcal{G}$ and so $D \leqslant Z(C)$. Also $D \leqslant G_{\bar{c} \tilde{f}}$ and so $[G, D] \leqslant M$. Then $[G, D,\langle a\rangle] \leqslant[M,\langle a\rangle]=1$. Also $[D,\langle a\rangle, G]=1$. Hence $[G,\langle a\rangle, D]=1$. Since $G=[G,\langle a\rangle] C$ and since $[D, C]=1$, we obtain $[D, G]=1$. This implies that $C_{G / Z(G)}(a) \in \mathscr{F}$ for all $a \in C_{A}(M)$. This in turn implies that $G / Z(G) \in \mathscr{F}$, contrary to $G \notin \mathcal{G}$. Hence $Z(G)=1$.

\section{If $B \leqslant A$ and $m(B) \geqslant 2$, then $C_{G}(B) \in \mathscr{F}$.}

Proof. Let $C=C_{G}(B)$ and $D=C_{\mathscr{f}}$. Let $b \in B^{\#}$. Then $C_{G}(b) \geqslant C$. Since $C_{G}(b) \in \mathcal{G}$,

$$
D \leqslant\left(C_{G}(b)\right)_{\mathscr{F}} \leqslant Z\left(C_{G}(b)\right) .
$$

Thus $\left[D, C_{G}(b)\right]=1$ for all $b \in B^{\#}$. Since $m(B) \geqslant 2, G=\left\langle C_{G}(b) \mid b \in B^{\#}\right\rangle$. Hence $[D, G]=1$. Since $Z(G)=1$, we obtain $D=1$ and so $C \in \mathscr{F}$.

4. $C_{G}(a) \in \mathscr{F}$ for all $a \in A^{\#}$.

Proof. Let $a \in A^{\#}$ and $H=C_{G}(a) . A$ has a subgroup $A_{1}$ such that $A=\langle a\rangle \times$ $A_{1}$. If $b \in A_{1}^{\#}$, we have $C_{H}(b)=C_{G}(\langle a, b\rangle) \in \mathscr{F}$ by (3). Since $m\left(A_{1}\right) \geqslant n(\mathscr{F})$ and since $C_{H}(b) \in \mathscr{F}$ for all $b \in A_{1}^{\#}, H$ must belong to $\mathscr{F}$.

\section{Contradiction.}

Proof. Since $C_{G}(a) \in \mathscr{F}$ for all $a \in A^{\#}$ and since $m(A)>n(\mathscr{F}), G \in \mathscr{F}$. But then $G / Z(G) \in \mathscr{F}$ and so $G \in \mathcal{G}$.

\subsection{Corollary. For $k \geqslant 0$, define $e_{k}$ by}

$$
e_{k}=\{G \mid G \text { nilpotent and } \operatorname{cl}(G) \leqslant k\} \text {. }
$$

Then $\bigodot_{k}$ is admissible and $n\left(\bigodot_{k}\right)=k+2$.

Proof. For $k=1$, this was proved in [7]. Now $e_{0}$ is the identity class and so $n\left(\bigodot_{0}\right)=2$. If $k \geqslant 1$, then

$$
e_{k}=\left\{G \mid G / Z(G) \in e_{k-1}\right\} .
$$

It now follows by induction on $k$ and by the theorem that $n\left(e_{k}\right) \leqslant k+2$. Example 2 in $\S 4$ shows that $n\left(\bigodot_{k}\right)$ is no smaller.

3.9. Corollary. Let $\mathbb{Q}$ denote the class of all cyclic groups. Then $\mathbb{Q}$ is admissible and $n(\mathbb{Q})=3$. 
Proof. $G \in \mathbb{Q}$ if, and only if, $G$ is abelian and $m(P) \leqslant 1$ for each Sylow subgroup $P$ in $G$. Putting 3.8 together with 2.12 and 2.8 yields $n(\mathbb{A}) \leqslant 3$. Example 3 in $\S 4$ shows that $n(\notin)$ is at least 3 .

The next result was first proved by Ward [15].

3.10 Corollary. If $\mathbb{\Psi}=\left\{G \mid G^{\prime}\right.$ is nilpotent $\}$, then $n(\mathbb{Q})=4$.

Proof. $\mathscr{U}=\{G \mid G / F(G)$ is abelian $\}$ and so $n(\mathbb{Q}) \leqslant 4$ using 3.8 together with 3.1 (with $\Lambda$ being the discrete partition). The last example in [16] shows that $n(\mathbb{Q}) \geqslant 4$.

The next result is useful in proving the admissibility of the class of supersolvable groups.

3.11. CoROllaRY. Let $n$ be a positive integer and let $\mathbb{Q}=\left\{G \mid G^{\prime}=1\right.$ and $x^{n}=1$

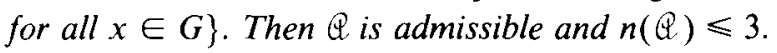

Proof. Suppose $A$ acts on $G,(|A|,|G|)=1, A$ is elementary abelian, $C_{G}(a)$ $\in Q$ for all $a \in A^{\#}$, and $m(A) \geqslant 3$. Then $G$ is abelian by 3.8. Since $G$ is generated by the subgroups $C_{G}(a)$ with $a \in A^{\#}$, we see that $x^{n}=1$ for all $x \in G$.

3.12. THEOREM. Let $\mathscr{F}$ be K-generated by $\{\mathscr{F}(p)\}$ where each non-empty $\mathscr{F}(p)$ is an admissible subgroup-closed formation. Assume further that $\{n(\mathscr{F}(p)) \mid \mathscr{F}(p) \neq$ $\varnothing\}$ has an upper bound. Then $\mathscr{F}$ is admissible and

$$
n(\mathscr{F}) \leqslant 1+\sup \{n(\mathscr{F}(p)) \mid \mathscr{F}(p) \neq \varnothing\} .
$$

Proof. Let $\pi=\{p \mid \mathscr{F}(p) \neq \varnothing\}$ and let $\mathscr{P}$ be the class of all $\pi$-groups. For $p \in \pi$, define $\mathcal{G}(p)$ by

$$
\mathcal{G}(p)=\left\{G \mid G / K_{p}(G) \in \mathscr{F}(p)\right\} .
$$

Then $n(\mathcal{G}(p)) \leqslant n(\mathscr{F}(p))+1$ by 3.1. Since $n(\mathscr{P}) \leqslant 2$, and since $\mathscr{F}=\mathscr{P} \cap$ $\cap_{p \in \pi} \mathcal{G}(p)$, the result now follows from 2.8 .

The next result was first proved in [8].

3.13. COROLlary. If $\delta$ is the class of all supersolvable groups, then $\delta$ is admissible and $n(\S)=4$.

Proof. For each prime $p$, let $\mathscr{T}(p)$ be the class of all groups $G$ such that $G$ is $p$-closed and a Hall $p^{\prime}$-subgroup of $G$ is abelian of exponent dividing $(p-1)$. It 
follows from $3.5,2.9$, and 3.10 , that $n(\mathscr{F}(p)) \leqslant 3$. Now it is straight forward to verify that $\{\mathscr{F}(p)\} K$-generates $\delta$. Hence $n(\delta) \leqslant 4$. The reverse inequality follows from an example in [8].

We now prove the necessary machinery to handle formations defined in terms of $\pi$-length.

3.14. LEMma. Let $\mathscr{F}$ be an admissible, subgroup-closed formation. Let $\mathcal{G}=\{G$ $\left.G / O_{\pi}(G) \in \mathscr{F}\right\}$. Then $\mathcal{G}$ is an admissible, subgroup-closed formation and $n(\mathcal{G}) \leqslant$ $n(\mathscr{F})+1$.

ProOF. It is straightforward to verify that $\mathcal{G}$ is a subgroup-closed formation. Assume now that $A$ acts on $G,(|A|,|G|)=1, A$ is elementary abelian, $C_{G}(a) \in \mathcal{G}$ for all $a \in A^{\#}$, and $m(A) \geqslant n(\widetilde{y})+1$. Assume that $G$ is a minimal example such that $G \notin \mathcal{G}$. Let $M$ be the $\mathcal{G}$-residual of $G$. Then $M \neq 1$ and every non-identity $A$-invariant normal subgroup of $G$ must contain $M$. In particular, since $G$ cannot belong to $\mathscr{F}, G_{\mathscr{f}} \geqslant M$. Since $G / M \in \mathcal{G}, G_{\mathscr{F}} / M \leqslant O_{\pi}(G / M)$.

Now $K_{\pi}\left(C_{G}(a)\right) \geqslant O_{\pi}\left(C_{G}(a)\right)$ and so $C_{G}(a) / K_{\pi}\left(C_{G}(a)\right) \in \mathscr{F}$ for all $a \in A^{\#}$. Then 3.3 implies that $G / K_{\pi}(G) \in \mathscr{F}$. Hence $G_{\mathscr{F}} \leqslant K_{\pi}(G)$ and so $K_{\pi}(G) \neq 1$. then $K_{\pi}(G) \geqslant M$. Now $G \notin \mathcal{G}$ and so $G / O_{\pi}(G) \notin \mathscr{F}$. It follows that $K_{\pi}(G)$ is not a $\pi$-group. Since $O_{\pi}(G)$ and $O_{\pi^{\prime}}(G)$ cannot both be different from the identity, we must have $O_{\pi}(G)=1$ and $O_{\pi^{\prime}}(G)=K_{\pi}(G)$. Then $G_{\tilde{F}}$ is a $\pi^{\prime}$-group.

If $a \in A^{\#}$, then $C_{G}(a) / O_{\pi}\left(C_{G}(a)\right) \in \mathscr{F}$. Hence $\left(C_{G}(a)\right)_{\mathscr{F}} \leqslant O_{\pi}\left(C_{G}(a)\right)$. Since $\sigma_{F}$ is subgroup-closed, $\left(C_{G}(a)\right)_{\tilde{F}} \leqslant G_{\widetilde{y}} \leqslant O_{\pi^{\prime}}(G)$. We now obtain $C_{G}(a) \in \mathscr{\mathscr { y }}$ for all $a \in A^{\#}$. But since $m(A)>n(\mathscr{F})$, this implies that $G \in \mathscr{F}$ and the proof is complete.

3.15. THEOREM. Let $\mathcal{F}$ be an admissible, subgroup-closed formation. Let $\mathcal{G}=\{G \mid$ $\left.G / O_{\pi^{\prime} \pi}(G) \in \mathscr{F}\right\}$. Then $\mathcal{G}$ is an admissible, subgroup-closed, saturated formation and $n(\mathcal{G}) \leqslant n(\mathscr{F})+2$.

Proof. Let $\mathcal{K}=\left\{G \mid G / O_{\pi}(G) \in \mathscr{F}\right\}$. Then $\mathcal{G}=\left\{G \mid G / O_{\pi^{\prime}}(G) \in \mathscr{K}\right\}$ and so 2 applications of 3.14 yield the result.

3.16. Corollary. Suppose $\pi$ is non-trivial and let $\mathscr{P}_{k}$ denote the class of all $\pi$-separable groups of $\pi$-length $\leqslant k$. Then $\mathscr{P}_{k}$ is admissible and $n\left(\mathscr{P}_{k}\right)=2 k+2$.

Proof. $\mathscr{P}_{0}$ is the class of all $\pi^{\prime}$-groups and so $n\left(\mathscr{P}_{0}\right)=2$. Now if $k \geqslant 1$, then $G \in \mathscr{P}_{k}$ if, and only if, $G / O_{\pi^{\prime} \pi}(G) \in \mathscr{P}_{k-1}$. Using the theorem and induction on $k$, we obtain $n\left(\mathscr{P}_{k}\right) \leqslant 2 k+2$ for all $k$. Example 4 in $\S 4$ demonstrates that $n\left(\mathscr{P}_{k}\right)$ cannot be smaller than $2 k+2$. 
3.17. THEOREM. Let $\mathscr{F}$ be locally defined by $\{\mathscr{F}(p)\}$ where each non-empty $\widetilde{F}(p)$ is an admissible, subgroup-closed formation. Assume further that $\{n(\mathscr{F}(p)) \mid$ $\widetilde{\mathcal{F}}(p) \neq \varnothing\}$ has an upper bound. Then $\mathscr{F}$ is admissible and $n(\mathscr{F}) \leqslant 2+$ $\sup \{n(\widetilde{\mathscr{F}}(p) \mid \widetilde{F}(p)) \neq \varnothing\}$.

Proof. The proof of 3.17 is identical with the proof of 3.12 except that $K_{p}(G)$ is replaced by $O_{p^{\prime} p}(G)$ and 3.16 is used instead of 3.1 .

Note that although each saturated formation is both locally defined and $K$-generated, we may not get as good a bound for $n(\mathscr{F})$ using 3.17 as compared with 3.12. For example, suppose $\mathscr{F}(p)$ is the class of all abelian groups of exponent dividing $p-1$. Then $n(\mathscr{F}(p))=3$ if $p>2$ and $\{\mathscr{F}(p)\}$ locally defines $\S$, the class of all supersolvable groups. Thus using 3.17 would yield $n(\delta) \leqslant 5$ which is weaker than $n(\mathcal{S}) \leqslant 4$, the result we obtained using 3.12 .

To show the necessity of requiring that $\{n(\mathscr{F}(p))\}$ has an upper bound and also to exhibit a subgroup-closed, saturated formation which is not admissible, we have the following result.

3.18. ThEOREM. Suppose $f(p)$ is a positive integer for each prime $p$. Define $\mathscr{F}$ by

$$
\mathscr{Y}=\left\{G \mid G \text { is solvable and } l_{p}(G) \leqslant f(p) \text { for all } p\right\} .
$$

Then $\mathscr{G}$ is a subgroup-closed saturated formation. $\mathscr{Y}$ is admissible if, and only if, $\{f(p) \mid p$ a prime $\}$ has an upper bound.

ProOF. of is certainly a subgroup-closed saturated formation and the only question is whether or not $\mathscr{F}$ is admissible. Now if $f(p) \leqslant N$ for all $p$, then $n(\vec{y}) \leqslant 2 N+2$ by 3.16 and 2.8 .

Now suppose that $\{f(p)\}$ has no upper bound. Let $n$ be any positive integer. Then there must be primes $p$ and $q$ such that

$$
\frac{n-1}{2}<f(p)<f(q) \text {. }
$$

Next let $m=2 f(p)+1$ and let $r$ be any prime distinct from $p$ and $q$. If $Q$ is an elementary abelian $r$-group of order $r^{m}$, then it follows from [7] that there is a $\{p, q\}$-group $G$ such that $A$ acts in a fixed-point-free manner on $G, l(G)=m$, and $l_{p}(G)=\left[\frac{1}{2}(m+1)\right]=f(p)+1$. Hence $G \notin \mathscr{F}$. Assume that $a \in A^{\#}$ and $C=C_{G}(a)$. Then $A /\langle a\rangle$ acts without fixed points on $C$. Hence $l(C) \leqslant m-1$ by [2]. It follows from this that

$$
l_{p}(C) \leqslant[m / 2]=f(p) \text { and } l_{q}(C) \leqslant[m / 2]=f(p) \leqslant f(q) .
$$

Therefore $C_{G}(a) \in \widetilde{F_{F}}$ for all $a \in A^{\#}$. Since $G \notin \widetilde{F}$ and $m(A)=m>n$, we see that $n(\widetilde{T})$ cannot be $\leqslant n$. Since $n$ was arbitrary, $\widetilde{\mathscr{F}}$ cannot be admissible. 


\section{Examples}

1. Let $\pi$ be non-trivial. Then there are primes $p$ and $q$ with $p \in \pi$ and $q \in \pi^{\prime}$. If $r$ is any prime distinct from $p$ and $q$, then [7] implies that there is a $\{p, q\}$-group $G$ such that $O_{p}(G)=1, l(G)=2$, and $G$ admits a fixed-point-free operator group $A$ which is an elementary abelian $r$-group of order $r^{2}$. Then $G$ is not $\pi$-closed but $C_{G}(a)$ has a fixed-point free operator group $A /\langle a\rangle$ of prime order. Thus $C_{G}(a)$ is nilpotent $[6,10.2 .1]$ and so $C_{G}(a)$ is $\pi$-closed for all $a \in A^{\#}$. This example justifies the equality in 3.5 .

2. Let $p$ and $q$ be primes with $p \equiv 1(\bmod q)$. Let $k$ be any positive integer and let $V$ be an elementary abelian $p$-group with $m(V)=k+1$. Let $H=\operatorname{Aut}(V)$, let $P$ be a Sylow $p$-subgroup of $H$, and let $G=V P$. If $N=N_{H}(P)$, then $N / P$ is the direct product of $(k+1)$ copies of a cyclic group of order $p-1$. Then $N$ must contain a subgroup $A$ such that $A$ is an elementary abelian $q$-group and $m(A)=$ $k+1$. Since $A$ normalizes $P, A$ will operate on $G$. I assert that $\operatorname{cl}\left(C_{G}(a)\right) \leqslant k$ for all $a \in A^{\#}$ while $\operatorname{cl}(G)>k$. This justifies the equality in 3.38 .

Now if $1 \leqslant n \leqslant k+1$, then it is easy to verify that

$$
|[V, \underbrace{P, P, \ldots, P}_{n}]|=p^{k+1-n} .
$$

It follows from this that $L_{k+1}(G) \neq 1$. Hence $\operatorname{cl}(G)>k$. (Actually, $\operatorname{cl}(G)=k+1$ but we don't need this.) Suppose $a \in A^{\#}, C=C_{G}(a), Q=C_{P}(a)$, and $U=C_{V}(a)$. Then $C=U Q$ and $L_{k+1}(Q)=1[10$, III.16.3] and so

$$
L_{k+1}(C)=[U, \underbrace{Q, Q, \ldots, Q}_{k}] \text {. }
$$

If $L_{k+1}(C) \neq 1$, then we would have

$$
U>[U, Q]>[U, Q, Q]>\cdots>[U, \underbrace{Q, \ldots, Q}_{k}]>1
$$

This would imply that

$$
|U| \geqslant p^{k+1}=|V|
$$

Since $1 \neq a \in \operatorname{Aut}(V)$, this is impossible. Hence $L_{k+1}(C)=1$ and so $\operatorname{cl}\left(C_{G}(a)\right)$ $\leqslant k$ for all $a \in A^{\#}$. 
3. Let $G$ be an elementary abelian group of order 9 with basis $\{x, y\}$. Let $A$ be an elementary abelian group of order 4 with generators $\{a, b\}$. Have $A$ operate on $G$ by $x^{a}=x^{-1}, y^{a}=y, x^{b}=x, y^{b}=y^{-1}$. Then $C_{G}(c)$ is cyclic for all $c \in A^{\#}$ but $G$ is not cyclic. Thus we have the example needed for 3.9.

4. If $\pi$ is non-trivial, then there exist primes $p$ and $q$ with $p \in \pi$ and $q$ in $\pi^{\prime}$. Let $r$ be any prime distinct from $p$ and $q$, let $k$ be any positive integer, and let $A$ be an elementary abelian $r$-group $A$ with $m(A)=2 k+1$. It follows from [7] that there is a $\{p, q\}$-group $G$ such that $A$ operates in a fixed-point-free manner on $G$, $O_{q}(G)=1$, and $l(G)=2 k+1$. Then $l(G)=l_{\pi}(G)+l_{\pi^{\prime}}(G)$ and $l_{\pi}(G) \geqslant l_{\pi^{\prime}}(G)$. Hence we must have $l_{\pi}(G)=k+1$. However, if $a \in A^{\#}$ and $C=C_{G}(a)$, then $A /\langle a\rangle$ acts in a fixed-point-free manner on $C$. It follows from [2] that $l(C) \leqslant 2 k$. But then

$$
l_{\pi}(C)=l_{p}(C) \leqslant\left[\frac{2 k+1}{2}\right]=k
$$

Hence $l_{\pi}\left(C_{G}(a)\right) \leqslant k$ for all $a \in A^{\#}$ but $l_{\pi}(G)>k$. This justifies the equality in 3.16 .

\section{References}

[1] M. Aschbacher and G. Seitz, 'On groups with a standard component known type'. Osaka Math.J. 13 (1976), 439-482.

[2] T. Berger, 'Nilpotent fixed point free automorphism groups of solvable groups', Math. Z. 131 (1973), 305-312.

[3] W. Feit and J. Thompson, 'Solvability of groups of odd order', Pacific J. Math. 13 (1963), $755-1029$.

[4] L. Finkelstein, 'Finite groups with a standard component of type $J_{4}$ '. Pacific J. Math. 71 (1977), 41-56.

[5] G. Glauberman, 'On solvable signalizer functors in finite groups', Proc. London Math. Soc. (3) 33 (1976), 1-27.

[6] D. Gorenstein, Finite groups (Harper and Row, New York, 1968).

[7] F. Gross, 'A note on fixed-point-free solvable operator groups', Proc. Amer. Math. Soc. 19 (1968), 1363-1365.

[8] F. Gross, 'Elementary abelian operator groups', Bull. Austral. Math. Soc. 7 (1972), 91-100.

[9] P. Hall, 'Theorems like Sylow's', Proc. London Math. Soc. (3) 6 (1956), 286-304.

[10] B. Huppert, Endliche Gruppen I (Springer, Berlin, 1967).

[11] W. Jonés, private communication.

[12] P. Schmid, 'Every saturated formation is a local formation', J. Algebra 51 (1978), 144-148.

[13] R. Steinberg, Lectures on Chevalley groups, (Yale University, 1967).

[14] J. Ward, 'On finite groups admitting automorphisms with nilpotent fixed-point group', Bull. Austral. Math. Soc. 5 (1971), 281-282. 
[15] J. Ward, 'On finite soluble groups and the fixed point groups of automorphisms'. Bull Austral. Math. Soc. 5 (1971), 375-378.

[16] J. Ward, 'Nilpotent signalizer functors on finite groups', Bull. Austral. Math. Soc. 9 (1973). $367-377$.

Department of Pure Mathematics

Australian National University

Canberra ACT 2600

Australia
Department of Mathematics

University of Utah

Salt Lake City Utah 84112

U.S.A. 